\title{
ON THE EFFECTS OF ACQUISITION PARAMETERS AND SURFACE PROPERTIES IN SEA OIL SEEP OBSERVATION BY MEANS OF HIGH-RESOLUTION SAR
}

\author{
F. Nunziata ${ }^{1}$, C.R. de Macedo ${ }^{1}$, A. Buono ${ }^{1}$, D. Velotto $^{2}$ and M. Migliaccio ${ }^{1}$ \\ ${ }^{1}$ Dipartimento di Ingegneria, Università degli Studi di Napoli Parthenope, Napoli, Italy \\ ${ }^{2}$ Remote Sensing Technology Institute, German Aerospace Center (DLR), 28199 Bremen, Germany
}

\begin{abstract}
In this study, a comprehensive analysis of $\mathrm{X}$-band backscattering from sea oil seeps is undertaken exploiting: 1) a twoscale scattering model to predict $\mathrm{vv}$-polarized sea surface backscattering with and without oil slicks under different wind speeds and incidence angles; 2) a large time-series of co-polarized dual-polarimetric Synthetic Aperture Radar imagery collected by the German TerraSAR-X mission over the well-known oil seep related to the Taylor Energy accident occurred in 2004 in the Gulf of Mexico.
\end{abstract}

Index Terms - Oil seeps, scattering models, SAR

\section{INTRODUCTION}

Sea oil slick observation using Synthetic Aperture Radar (SAR) imagery is a quite mature application whose performance is significantly affected by the amount of scattering information available. This latter, at once, depends on both sensor's and environmental parameters, e.g.; polarimetric mode, incidence angle, noise equivalent sigma zero (NESZ), sea state conditions and oil's damping properties.

Although the key role played by polarimetric information in both discriminating actual oils from look-alikes and providing rough information on the damping properties of the surfactant is well-established [1], the large availability of single-polarization SAR imagery covering larger areas makes operational sea oil slick observation mainly based on singlepolarization intensity SAR acquisitions. Hence, in this study, a comprehensive analysis of the backscattering from sea oil slicks is undertaken exploiting a two-scale scattering model and a large time-series of coherent co-polarized SAR scenese collected by the German TerraSAR-X satellite mission over an oil slick generated by the certified oil seep observed in Gulf of Mexico since the Taylor Energy accident in 2004, see Fig. 1.

The proposed analysis aims at addressing slick-free and oil-covered sea surface backscattering at different incidence angles and wind regimes and, in addition, at evaluating the

This study is partially supported by Università degli Studi di Napoli Parthenope under the project ID DING 202 and by European Space Agency under the Dragon-4 project ID 32235. influence of noise. In fact, although the influence of those factors on SAR-based sea oil slick detection has been the objective of several investigations ([2] - [6]), such experiments exploited only a limited number of SAR images that are typically collected by different SAR sensors and/or in different locations, with a narrow range of incidence angles and sea state conditions. Hence, to the best of our knowledge, there is no study aimed at addressing the role of the above mentioned parameters using a time series of SAR imagery collected over a well-known oil seepage.

Hence, in this study, a time-series that consists of 42 TSX StripMap SAR scenes collected over the same test site with different polarizations, incidence angles and meteo-marine conditions, is exploited (see detailed information listed in Table 1). The analysis is carried out using both scattering model predictions (obtained using a two-scale backscattering model) and experimental features extracted from actual SAR measurements.

\section{THEORETICAL RATIONALE}

In this section, the scattering model used to predict slickfree and oil-covered sea surface backscattering is briefly described.

Sea surface scattering can be well-described, under low-tomoderate wind regimes, i. e., $2 \mathrm{~m} / \mathrm{s}-12 \mathrm{~m} / \mathrm{s}$, and at intermediate incidence angles, i. e., $20^{\circ}-60^{\circ}$, by two-scale models [7]. The latter assume the total normalized radar cross section (NRCS), $\sigma^{o}$, as the sum of two contributions:

$$
\sigma^{o}=\sigma_{0}^{o}+\sigma_{1}^{o}
$$

where $\sigma_{0}^{o}$ is the zero-th order term that accounts for the scattering of the long-wave part of the sea surface spectrum and it is modeled by the Geometric Optic (GO) high-frequency approximation. The $\sigma_{1}^{o}$ term is related to the backscattering from the small-scale ripples modulated by the long-wave structure (tilted-Bragg scattering). The analytical expression of the two contributions is shown in eq. (2) on the top of the next page, where $t$ and $r$ indicate the transmitted and received polarization, respectively, $\tau_{s p}$ is the angle between the local normal and the vertical, $R_{r t, e f f}$ is the local incidence 


$$
\sigma_{r t}^{o}=\frac{\pi}{\cos ^{2}\left(\tau_{s p}\right)}\left|R_{r t, e f f}\right|^{2} T_{s l}\left(\alpha_{s p}, \beta_{s p}\right)+\frac{k^{2}}{4 \pi\left(\nu_{z} / k\right)^{2}} \iint\left|H_{r t}\left(K_{x}^{\prime}, K_{y}^{\prime}\right)\right|^{2} \psi_{R}\left(K_{x}, K_{y}\right) T_{s l}\left(K_{x}^{\prime}, K_{y}^{\prime}\right) d K_{x} d K_{y}
$$

angle-dependent effective Fresnel reflection coefficient that accounts for the reduction of the specular-like backscattering due to the small-scale roughness on the tangent plane, $T_{s l}(\cdot)$ is the slope distribution evaluated at the specular points $\alpha_{s p}$ and $\beta_{s p}, k$ is the electromagnetic wavenumber, $H_{r t}(\cdot)$ is the surface field function which depends on incidence angle $\theta$ and the sea dielectric constant $\epsilon_{w}$, and $\psi_{R}$ is the normalized ripple spectrum [8, 9].

To predict oil-covered sea surface backscattering, two oilrelated effects must be included in the model (see eq. (2)): i) the damping of the small gravity waves that depends on the visco-elastic properties of the surfactant through its rheological parameters; ii) reduction of the energy input from the wind to the waves. The first effect is described by the Marangoni viscous damping coefficient $[10,11]$ :

$$
C(K,|E|, \varphi)=\frac{1+X(\cos \varphi-\sin \varphi)+X Y-Y \sin \varphi}{1+2 X(\cos \varphi-\sin \varphi)+2 X^{2}}
$$

where $X$ and $Y$ are empirical coefficients that depend on the rheological parameters of the oil, i. e., its complex dilatational coefficient (modulus $|E|$ and phase $\varphi$ ), and on dynamic viscosity and density of the seawater.

The second effect is driven by the friction velocity that, with respect to the slick-free sea surface case $\left(u_{*}\right)$ is reduced using a penalty factor $\beta[7,11]$

$$
u_{* o}=\beta u_{*}
$$

The combination of the two effects results in a full-range sea surface spectrum that changes in both its short-wave (damping and reduction of the friction velocity) and long-wave part (reduction of the friction velocity). In addition the presence of an oil slick, reducing the sea surface spectrum, also affects the distribution of the surface slopes since its variances are related to the sea surface spectrum.

\section{EXPERIMENTS}

The first experiment consists of randomly sampling 1000 pixels over the slick-free and oil-covered Region of Interests (ROIs) excerpted over the whole TSX data set, for each SAR scene. Data-points related to $\sigma_{v v}^{o}$ are depicted in Fig. 2 where they are grouped into three classes according to their mean incidence angles: $\theta=23^{\circ}, 32^{\circ}, 43^{\circ}$. Note that the NESZ curve, evaluated for each SAR scene and interpolated over the incidence angles, is also shown. When dealing with slick-free sea surface, $\sigma_{v v}^{o}$ decreases significantly $(\approx 7 \mathrm{~dB})$ when $\theta$ moves from $26^{\circ}$ to $34^{\circ}$. This is physically explained by the fact that, according to the two-scale scattering model, the dominant scattering mechanism at $\theta=26^{\circ}$ is the specular-like

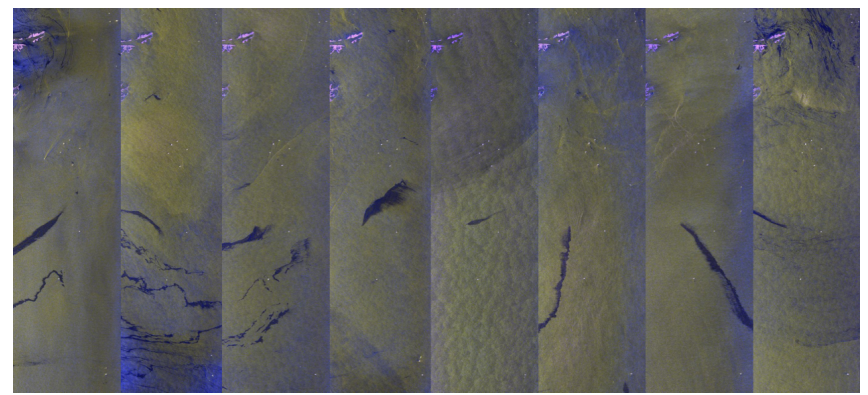

Fig. 1. False color RGB images relevant to the HH-VV TSX SAR data set collected in the Gulf of Mexico. Oil seep signatures over the ocean are clearly visible as dark patches, whose size, position, orientation and shape change in time.

Table 1. General overview on the SAR data set.

\begin{tabular}{c|c}
\hline \hline Acquisitions & $42 \mathrm{X}$-band TSX SAR scenes \\
\hline Imaging mode & HH-VV StripMap \\
\hline Acquisition period & July, $2011-$ April, 2016 \\
\hline Study area & Gulf of Mexico $\left(29^{\circ} \mathrm{N}, 89^{\circ} \mathrm{W}\right)$ \\
\hline Spatial resolution & $1.2 \mathrm{~m} \times 6.6 \mathrm{~m}($ slant range $\times$ azimuth $)$ \\
\hline$\theta$ range & $24^{\circ}-44^{\circ}$ \\
\hline Wind speed range & $1.8 \mathrm{~m} / \mathrm{s}-8.4 \mathrm{~m} / \mathrm{s}$ \\
\hline NESZ range & $-20.9 \mathrm{~dB}--23.0 \mathrm{~dB}$ \\
\hline \hline
\end{tabular}

scattering, that is well-modeled by the GO solution [7]. According to GO, the $\sigma_{v v}^{o}$ decreases significantly with $\theta$. At $\theta>$ $34^{\circ}$, the dominant scattering contribution is well-modeled by tilted-Bragg scattering that predicts a smoother decrease with $\theta$. However, it can be noted that there are few data-points that are very close and partly fall below NESZ at $\theta=43^{\circ}$ ). This analysis witnesses that, even for slick-free sea surface, at larger $\theta$ the backscattered signal is partially contaminated by noise. When dealing with oil-covered sea surface, datapoints are always below the corresponding slick-free sea surface ones, as expected. Even in this case, $\sigma_{v v}^{o}$ decreases with respect to $\theta$, although in a smoother way if compared to the slick-free backscattering. The oil/sea separation decreases with $\theta$, with the two backscattering values being almost overlapped at $\theta=43^{\circ}$. NESZ starts affecting oil-covered sea surface backscattering even at lower incidence angles, i. e. vv-polarized X-band SAR measurements over oil slicks are severely contaminated by noise since $\theta=34^{\circ}$ (most of the data-points lie below NESZ).

The second experiment aims at contrasting $\sigma_{v v}^{o}$ predicted by the two-scale model (see simulation parameters listed in Table 2) with the data-points measured within the slick-free 
Table 2. Parameters used for the two-scale backscattering model simulations.

\begin{tabular}{c|c}
\hline \hline Parameter & Value (Unit) \\
\hline Radar frequency & $9.6(\mathrm{GHz})$ \\
\hline Radar polarization & $\mathrm{VV}$ \\
\hline AOI range & $25-50\left(^{\circ}\right)$ \\
\hline Fetch & $500\left(\mathrm{~km}^{2}\right)$ \\
\hline$\epsilon_{w}$ & $58.19+\mathrm{j} 33.13[12]$ \\
\hline$|E|$ & $0.0225\left(\mathrm{Nm}^{-1}\right)$ \\
\hline$\varphi$ & $-175\left(^{\circ}\right)$ \\
\hline$\beta$ & 0.3 \\
\hline$\epsilon_{o}$ & $2.10+\mathrm{j} 0.02[13]$ \\
\hline \hline
\end{tabular}

ROI, see Fig. 3. It can be noted that model predictions well fit the measured data-points along the whole range of incidence angles.

The third experiment consists of contrasting oil-covered $\sigma_{v v}^{o}$ with data-points measured over the oil-covered ROI, see Fig. 4 and simulation parameters of Table 2 . It can be observed that model predictions do not fit the measured $\sigma_{v v}^{o}$ values at all the incidence angles. A fairly good agreement is obtained at lower incidence angles $\left(\theta \leq 34^{\circ}\right)$, while at $\theta=43^{\circ}$ there is no agreement. This is likely due to the fact that at larger incidence angle measurements are significantly corrupted by noise.

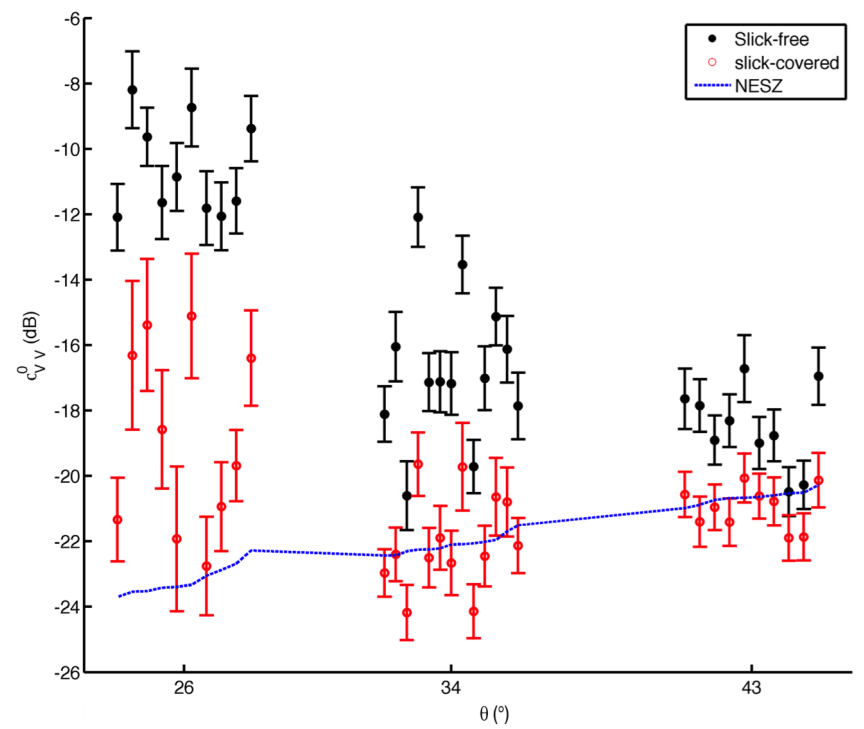

Fig. 2. $\sigma_{v v}^{o}$ evaluated using 1000 samples belonging to a slick-free (black) and oil-covered (red) ROI excerpted from the whole TSX data set. Note that the NESZ profile is also depicted, in blue.

\section{CONCLUSIONS}

In this study, sea surface backscattering with and without oil slicks is analyzed using a large data set of X-band SAR imagery collected over a well-known oil seep in a broad range of incidence angles and sea state conditions. Experimental results are contrasted with model predictions obtained using a two-scale scattering model.

The main outcomes can be summarized as follows:

- The predicted slick-free sea surface backscattering well fits measurements at all the incidence angles.

- The predicted oil-covered backscattering fairly agrees with measurements at lower incidence angles.

- TSX NESZ significantly limits measurements collected at larger incidence angles.

\section{ACKNOWLEDGMENTS}

We thank Prof. P. Sobieski who provided the sofware code used to predict slick-free sea surface backscattering. We also thank the German Space Agency (DLR) for providing the TerraSAR-X SAR data under the project ID AO OCE1045, and the National Oceanic and Atmospheric Administration (NOAA) for providing buoy data free of charge.

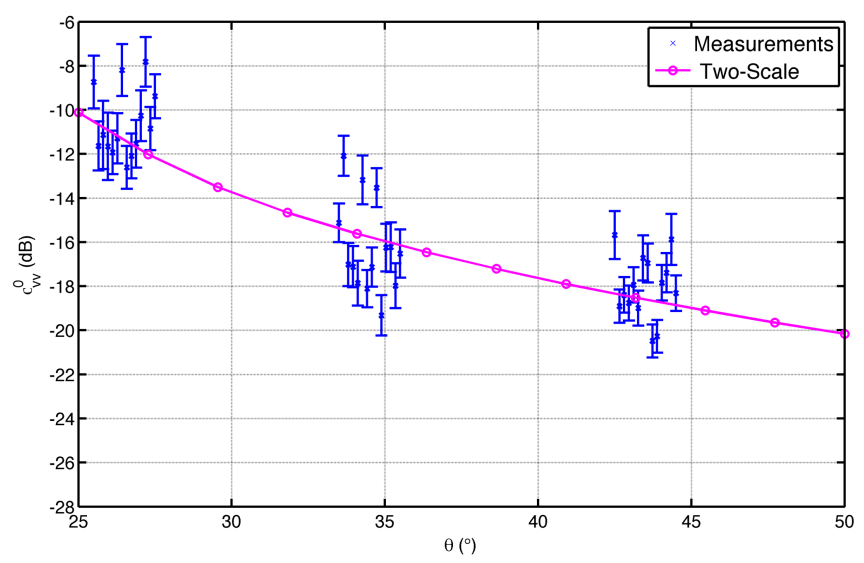

Fig. 3. Data-points related to $\sigma_{v v}^{o}$ evaluated over the slickfree sea surface ROI using the whole data set (blue markers) and slick-free $\sigma_{v v}^{o}$ predicted according to the two-scale model (magenta). 


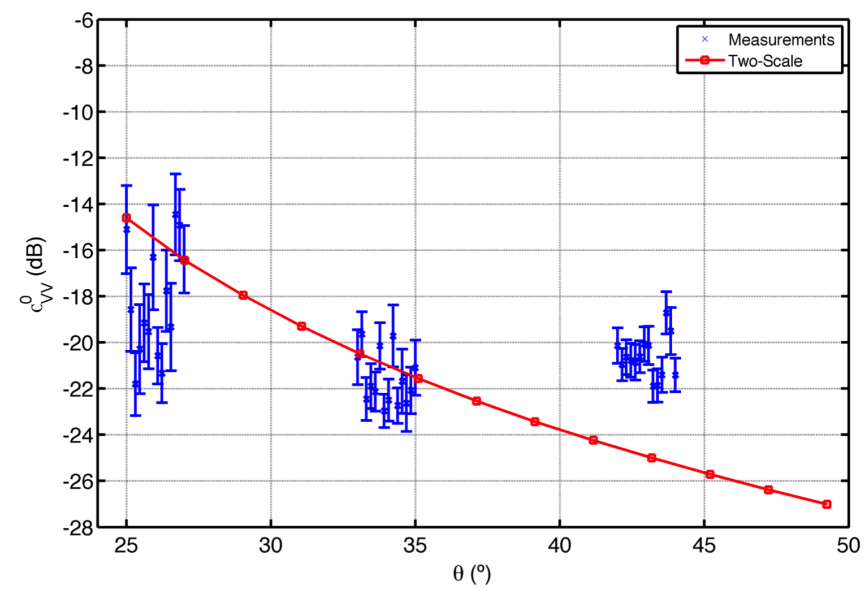

Fig. 4. Data-points related to $\sigma_{v v}^{o}$ evaluated over the oilcovered ROI using the whole data set (blue markers) and oilcovered $\sigma_{v v}^{o}$ predicted according to the two-scale model (magenta).

\section{REFERENCES}

[1] M. Migliaccio, F. Nunziata, and A. Buono, "SAR polarimetry for sea oil slick observation," Int. J. Remote Sens., vol. 36, no. 12, pp. 3243-3273, 2015.

[2] M. Gade, W. Alpers, H. Huhnerfuss, H. Masuko, and T. Kobayashi, "Imaging of biogenic and anthropogenic ocean surface films by the multifrequency/multipolarization SIR-C/X-SAR," J. Phys. Res., vol. 103, no. C9, pp. 18851-18866, 1998.

[3] V. Wismann, M. Gade, W. Alpers, and H. Huhnerfuss, "Radar signatures of marine mineral oil spills measured by an airborne multi-frequency radar," Int. J. Remote Sens., vol. 19, no. 18, pp. 3607-3623, 1998.

[4] B. Minchew, C.E. Jones, and B. Holt, "Polarimetric Analysis of Backscatter From the Deepwater Horizon Oil Spill Using L-Band Synthetic Aperture Radar," IEEE Trans. Geosci. Remote Sens., vol. 50, no. 10, pp. 3812-3830, 2012.

[5] D. Mera, V. Bolon-Canedo, J.M. Cotos, and A. AlonsoBetanzos, "On the use of feature selection to improve the detection of sea oil spills in SAR images," Comput. Geosci., vol. 100, no. C, pp. 166-178, 2017.

[6] S. Skrunes, C. Brekke, T. Eltoft, and V. Kudryavtsev, "Comparing near-coincident $\mathrm{C}$ - and X-band SAR acquisitions of marine oil spills," IEEE Trans. Geosci. Remote Sens., vol. 53, no. 4, pp. 1958-1975, 2015.

[7] F. Nunziata, P. Sobieski, and M. Migliaccio, "The twoscale BPM scattering model for sea biogenic slicks con- trast," IEEE Trans. Geosci. Remote Sens., vol. 47, n. 8, pp. 1949-1956, 2009.

[8] A. Guissard, P. Sobieski, and C. Baufays, "A unified approach to bistatic scattering for active and passive remote sensing of rough ocean surfaces," Trends Geophys. Res., vol. 1, pp. 43-68, 1992.

[9] S.A. Boukabara, L. Eymard, C. Guillou, D. Lemaire, P. Sobieski, and A. Guissard, "Development of a modified two-scale electromagnetic model simulating both active and passive microwave measurements: comparison to data remotely sensed over the ocean," Radio Sci., vol. 37, no. 4, pp. 16-1-16-11, 2002.

[10] P. Lombardini, B. Fiscella, P. Trivero, C. Cappa, and W. Garrett, "Modulation of the spectra of short gravity waves by sea surface films: Slick detection and characterization with a microwave probe," J. Atmos. Ocean. Technol., vol. 6, no. 6, pp. 882-890, 1989.

[11] A. Montuori, F. Nunziata, M. Migliaccio, and P. Sobieski, "X-band two-scale sea surface scattering model to predict the contrast due to an oil slick," IEEE J. Sel. Topics Appl. Earth Observ. Remote Sens., vol. 13, no. 11, pp. 4970-4978, 2016.

[12] W. Ellison, A. Balana, G. Delbos, K. Lamkaouchi, L. Eymard, C. Guillou, and C. Prigent, "New permittivity measurements of seawater," Radio Sci., vol. 33, no. 3, pp. 639-648, 1998.

[13] T. Friiso, Y. Schildberg, O. Rambeau, T. Tjomsland, H. Fordedal, and J. Sjoblom, "Complex permittivity of crude oils and solutions of heavy crude oil fractions," $J$. Disp. Sci. Techn., vol. 19, no. 1, pp. 93-126, 1998. 